\title{
Carbon Abundances in the Galactic Thin and Thick Disks
}

\author{
T. Bensby ${ }^{1} \&$ S. Feltzing ${ }^{2}$ \\ ${ }^{1}$ Department of Astronomy, 830 Dennison Building, University of Michigan, \\ Ann Arbor, MI 48109-1042, USA (email: tbensby@umich.edu) \\ ${ }^{2}$ Lund Observatory, Box 43, 22100 Lund, Sweden (email: sofia@astro.lu.se)
}

\begin{abstract}
Although carbon is, together with oxygen and nitrogen, one of the most important elements in the study of galactic chemical evolution its production sites are still poorly known and have been much debated (see e.g. Gustafsson et al. 1999; Chiappini et al. 2003). To trace the origin and evolution of carbon we have determined carbon abundances from the forbidden [C I] line at $8727 \AA$ and made comparisons to oxygen abundances from the forbidden [O I] line at 6300 $\AA$ in a sample of 51 nearby $\mathrm{F}$ and $\mathrm{G}$ dwarf stars. These data and the fact that the forbidden [C I] and $[\mathrm{O}$ I] lines are very robust abundance indicators (they are essentially insensitive to deviations from LTE and uncertainties in the stellar parameters, see, e.g., Gustafsson et al. 1999; Asplund et al. 2005) enable us to very accurately measure the $\mathrm{C} / \mathrm{O}$ ratio as well as individual $\mathrm{C}$ and $\mathrm{O}$ abundances. Our first results indicate that the time-scale for the main source that contribute to the carbon enrichment of the interstellar medium operate on the same time-scale as those that contribute to the iron enrichment (and can possibly be AGB stars...)
\end{abstract}

Keywords. Stars: abundances, stars: kinematics, Galaxy: abundances, Galaxy: disk

\section{Observations, data reduction, and abundance analysis}

Spectra for 16 thick disk stars and 35 thin disk stars (based on kinematical selection criteria, see Bensby et al. 2003, 2005) were obtained during six nights in September 2004 with the CES spectrograph on the ESO-3.6m telescope on La Silla. The spectra have a very high resolution of $R \sim 220000$ and high signal-to-noise ratios of $S / N \gtrsim 300$.

Carbon abundances were determined by comparing synthetic spectra (based on 1-D, plane parallel, LTE stellar atmosphere models that were calculated with the Uppsala MARCS code, Gustafsson 1975; Edvardsson 1993) to the observed spectra. The method used for the analysis is the same as that used for the forbidden oxygen line at $6300 \AA$ (Bensby et al. 2004). Stellar parameters were taken from Bensby et al. (2003, 2005). When synthesising the $[\mathrm{C} \mathrm{I}]$ line a blending Fe I line was taken into account (e.g., Lambert \& Swings 1967). Our analysis of the solar spectrum gives a carbon abundance in the Sun of $\log \epsilon(\mathrm{C})=8.41$, in good agreement with Allende Prieto et al. (2002) and Asplund et al. (2005) who determined a solar carbon abundance of 8.39 using 3D models.

\section{Results and discussion}

Our first results regarding carbon abundances in the Galactic thin and thick disks are (Fig. 1): (1) While the $[\mathrm{O} / \mathrm{Fe}]$ vs $[\mathrm{Fe} / \mathrm{H}]$ trends separate well for the thin and thick disk samples, they are merged for $[\mathrm{C} / \mathrm{Fe}]$ vs $[\mathrm{Fe} / \mathrm{H}]$ and are mainly flat; (2) The two kinematic samples separate well (as expected) in the $[\mathrm{C} / \mathrm{O}]$ vs $[\mathrm{O} / \mathrm{H}]$ plot; $(\mathbf{3})$ The thin disk sample in the same plot have a nearly constant $[\mathrm{C} / \mathrm{O}]$ ratio; (4) The thick disk sample first shows 

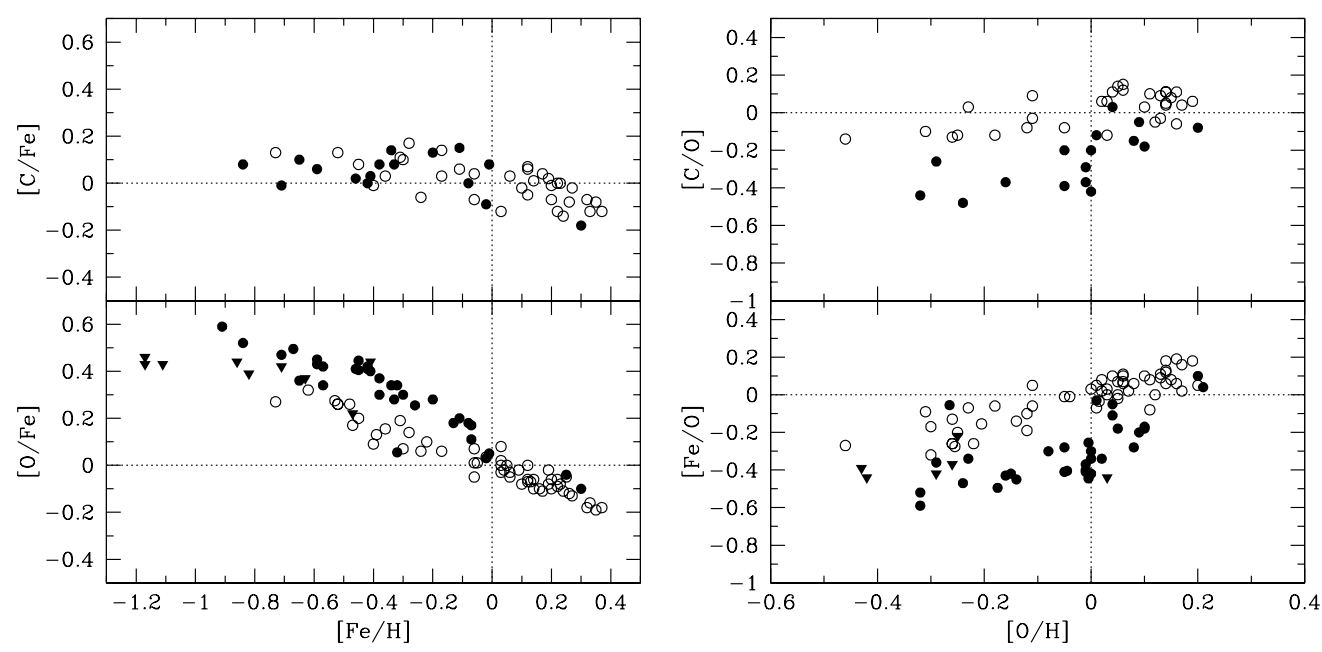

Figure 1. Our carbon trends relative to iron and relative to oxygen. For comparison we also show the $[\mathrm{O} / \mathrm{Fe}]$ vs $[\mathrm{Fe} / \mathrm{H}]$ and $[\mathrm{Fe} / \mathrm{O}]$ vs $[\mathrm{O} / \mathrm{H}]$ trends (Bensby et al. 2004, 2005). Thin and thick disk stars are marked by open and filled symbols, respectively. Filled triangles in the oxygen plots denote thick disks stars from Nissen et al. (2002).

a flat trend of $[\mathrm{C} / \mathrm{O}]$ vs $[\mathrm{O} / \mathrm{H}]$ for $[\mathrm{O} / \mathrm{H}]<0$ where $\mathrm{C}$ is depleted relative to $\mathrm{O}$ such that $[\mathrm{C} / \mathrm{O}]=-0.3$ to -0.4 dex but at $[\mathrm{O} / \mathrm{H}]=0$ the trend rises (sharply) to solar values.

A preliminary interpretation of these results is as follows: (I) In the thin disk the sources for $\mathrm{O}$ (SN II) and $\mathrm{C}$ (debated) are tuned such that a flat trend is created; (II) In the thick disk the processes are finely tuned up and until $[\mathrm{O} / \mathrm{H}]=0$. At $[\mathrm{O} / \mathrm{H}]=0$ a new source of $\mathrm{C}$ gets active (or a source of $\mathrm{O}$ drops out); (III) Studying the $[\mathrm{O} / \mathrm{Fe}$ ] vs $[\mathrm{Fe} / \mathrm{H}]$ trend we find that at $[\mathrm{Fe} / \mathrm{H}] \simeq-0.45 \mathrm{SN}$ Ia sets in (hence the appearance of the "knee" in the abundance plot). For $[\mathrm{C} / \mathrm{Fe}]$ this does not happen. This means that the time-scale for the population that contributes $\mathrm{C}$ operates on the same time-scale as SN Ia; (IV) These results could be exploited to derive an independent measure of the $\mathrm{SN}$ Ia lifetimes (given an understanding of the sources of C).

\section{Acknowledgements}

Thomas Bensby acknowledges support from the National Science Foundation, grant AST-0448900. Sofia Feltzing is a Royal Swedish Academy Research Fellow supported by a grant from the Knut and Alice Wallenberg Foundation.

\section{References}

Allende Prieto C., Lambert D.L., \& Asplund M., 2002, ApJ, 573, L137

Asplund M., Grevesse N., Sauval A.J., Allende Prieto C., \& Blomme R., 2005, A $\& A$, 417, 751

Bensby, T., Feltzing, S., \& Lundström, I., 2003, A\&A, 410, 527

Bensby, T., Feltzing, S., \& Lundström, I., 2004, A\&A, 415, 155

Bensby, T., Feltzing, S., Lundström, I., \& Ilyin, I., 2005, A\& $A$, 433, 185

Chiappini, C., Matteucci, F., \& Romano, D., 2003, MNRAS, 339, 63

Edvardsson, B., Andersen, J., Gustafsson, B. et al., 1993, A\& A, 275, 101

Gustafsson, B., Bell, R.A., Eriksson, K., \& Nordlund, Å., 1975, A\& $A, 42,407$

Gustafsson, B., Karlsson, T., Olsson, E., Edvardsson, B., \& Ryde, F., 1999, A\&3A, 342, 426

Lambert, D.L., \& Swings, J.P., 1967, Solar Physics, 2. 34

Nissen, P.E., Primas, F., Asplund, M., \& Lambert, D.L., 2002, A\&A, 390, 235 